\title{
A Comprehensive Convolutional Neural Network Survey to Detect Glaucoma Disease
}

\author{
Afia Zafar $\left(D,{ }^{1}\right.$ Muhammad Aamir $\left(D,{ }^{1,2}\right.$ Nazri Mohd Nawi $(D),{ }^{1}$ Sikandar Ali $(D), 3$ \\ Mujtaba Husnain $\mathbb{D}^{4},{ }^{4}$ and Ali Samad $\mathbb{D}^{4}$ \\ ${ }^{1}$ Faculty of Computer Science and Information Technology, Universiti Tun Hussein Onn Malaysia, Parit Raja, Malaysia \\ ${ }^{2}$ School of Electronics, Computing and Mathematics, University of Derby, Derby, UK \\ ${ }^{3}$ Department of Information Technology, The University of Haripur, Haripur, Khyber Pakhtunkhwa 22620, Pakistan \\ ${ }^{4}$ Department of Computer Sciences, Faculty of Computing, The Islamia University Bahawalpur, Bahawalpur 63100, Pakistan
}

Correspondence should be addressed to Sikandar Ali; sikandar@uoh.edu.pk

Received 10 December 2021; Accepted 13 January 2022; Published 1 February 2022

Academic Editor: Hasan Ali Khattak

Copyright (c) 2022 Afia Zafar et al. This is an open access article distributed under the Creative Commons Attribution License, which permits unrestricted use, distribution, and reproduction in any medium, provided the original work is properly cited.

Due to recent advances in computer technology and the accessibility of large datasets, deep learning has become at the forefront of artificial intelligence and on various tasks, especially those related to image classification and modelling, its performance is often equal to or even better than human appreciation. Ophthalmology has always been in an ideal position to employ one of CNN's most popular deep learning algorithms to evaluate vast volumes of data from these tests since it is one of the health practices that focuses extensively on imaging. Glaucoma is one among the situations in which CNN can benefit from the enormous quantity of data collected by tests that assess the anatomy as well as function of the optic nerve and macula. We discussed the recommended use of $\mathrm{CNN}$ algorithm for specific glaucoma scenarios, such as fundus photography screening and diagnosis and detection of the course of glaucoma through OCT imaging modality. The purpose of this article is not only to critically examine and discuss the latest applications of CNN models in glaucoma but also to focus on the challenges associated with developing such models for screening, diagnosis, and progress detection. After a brief overview of the clinical practices and their comparison with conventional clinical methods, we discussed training and validation of CNN algorithm and how it was developed and why it is particularly suitable for glaucoma. The following features make our contribution worthwhile and unique among the reviews of similar kind: (i) our review classifies the existing literature with respect to detection of the glaucoma disease using conventional and nonconventional approaches; (ii) it covers a very different outlook of the glaucoma disease by providing in-depth discussions of the existing works at different granularity levels, that is, from primary to mediatory to the severe level; (iii) this state-of-the-art review covers each article in the following dimensions: the computer-based approach to tomographic model; analysis of different datasets; and summarizing the literature review in a disciplined way by mentioning the research gap concluded with discussion on future work.

\section{Background}

According to a study by the World Health Organization, 64.3 million people worldwide are affected by this disease and it is estimated that the disease will grow to 80 million in 2020 and to 111.8 million in 2040. These figures are expected to increase by 2050 . For example, the number of blind people in Canada in 2013 was approximately 500,000 and it is estimated that 50,000 people will become blind each year [1]. Glaucoma is one of the main diseases that cause blindness.
Treating blindness and compensating for an individual's loss of ability to find meaningful work is a costly expense for individuals and for society. For example, in the UK, the burden of vision loss and blindness has increased from around 143,600 to over 154,600 disability-adjusted life years, while the disability-adjusted life years in the UK for various reasons have declined. In 2013, the total economic cost of vision loss and blindness in the $\mathrm{UK}$ was estimated to be between $\$ 2.4$ billion and $\$ 35.5$ billion [2]. The quantity of glaucoma patients within the United States is predicated to 
increase from 2.7 million in 2010 to 6.3 million in 2050 and by the end of 2020, the amount of glaucoma sufferers aged 40 to 80 was estimated to increase to 64.3 million and by 2040 to 111.8 million [3]. The profile of glaucoma patients in the coming decades is depicted in Figure 1.

\section{Introduction}

Glaucoma is the primary vision impairment diagnosed around the world. The main cause of glaucoma is the slow death of retinal ganglion cells (RGC) and increased intraocular pressure in the eye. This happens due to the increased mass of fluid in the eyeball. A raised bulge in the eye can damage nerve bundles (axons) in the nerve fiber layer of the retina $(\mathrm{RNF})$. It starts with a negligible problem and the breakdown of the neural chain as shown in Figure 2 below. These malformations are so trivial that they are generally incomprehensible in the early stages of glaucoma. The most common cause of visual impairment and possible blindness is that glaucoma is difficult to diagnose and treat early on. In Singapore, around $90 \%$ of glaucoma patients are unaware of their condition. In Australia, almost $50 \%$ of glaucoma patients are still unaware of their condition. In the United States, approximately 4 million people have experienced the side effects of this disease, and half of them are conscious of their disease again. According to a seminar by Dr. Mirza Shafiq, almost half of the 1.8 million glaucoma patients in Pakistan are permanently blind, unfortunately $2 \%$ of them are children $[6,7]$. Aside from early detection and treatment, there is no cure for glaucoma [8]. The high proportion of undiagnosed patients indicates the need for major changes to existing diagnostic procedures. Early detection depends on manual observation by the ophthalmologist. This is expensive and can go wrong. This is because not all ophthalmologists have the necessary experience to correctly diagnose glaucoma. Subsequently, most of the patients were either undiagnosed or misdiagnosed. Therefore, glaucoma can progress and cannot be treated until it is discovered. So, it is very important to develop automated tools that will aid in the early detection of glaucoma as it will have a positive impact on people and the economy. Therefore, it is very important to develop automated methods for the early detection of glaucoma. These methods will use the experience of various ophthalmologists to get reliable results.

2.1. Types of Glaucoma. Various sorts of glaucoma are available all over the world, while the two significant categories of glaucoma are open-angle and angle-closure glaucoma, which can be answerable for development in intraocular pressure. Open-angle glaucoma [9] that is also known as primary open-angle glaucoma (POAG) is a common type of glaucoma, which appeared to be the main leading cause of glaucoma spread around the world. According to the Glaucoma Research Centers, almost more than $90 \%$ cases are because of open-angle glaucoma. Primary open-angle glaucoma is a cutting-edge optic neuropathy and, perhaps, the most widely recognized type of glaucoma. Angle-closure glaucoma [10], which is also acknowledged as closed angle, occurs due to complete aqueous fluid blockage leading to a critical rise of IOP. The pressure rises so speedily that it may affect the vision in a swift manner, and it demands instantaneous medical attention.

\section{Related Work}

Glaucoma is characteristically found out by means of an affected person after an extended period of disease progression. Over the years, the same procedures have been used by the ophthalmologists to diagnose the glaucoma. Those procedures depend exclusively on ophthalmologists' manual interpretations and therefore $80 \%$ of glaucoma cases are not detected at their early stage. Consequently, it is crucial to process everyday eye tests on the way to find the symptoms of glaucoma and to deal with symptoms as early as possible.

\subsection{Conventional Clinical Methods to Detect Glaucoma.} Tonometer instrument [11] is used to measure IOP in the retina. Currently, numerous techniques of tonometry are in practice, among which Goldmann is widely adopted. Due to irregular cornea thickness, tonometry will not be able to produce proper measurements. To overcome this issue, Pachymetry test is getting used by ophthalmologists. It helps to regulate thickness cornea, as it is significant because the patient with thinner cornea and authentic IOP may be underestimated and with thicker cornea may be overestimated. If ophthalmologists are not sure about glaucoma identification even after performing tonometry and pachymetry, then other tests (i.e., gonioscopy, perimetry, and ophthalmoscopy) are conducted [12]. Perimetry is used to check patient vision by measuring the light sensitivity by detecting targets. However, this test is time-consuming because it may take three attempts before an accuracy is obtained. Thus, results may be compromised, and the accuracy of this method remains questioned. To get the clean and greater correct result and to determine the thickness of RNFL, ophthalmologists are going for 3D imaging modalities. However, these tools are not available everywhere as they are expensive and furthermore, clinics, in particular, practice these procedures even though entirely different tests specify a conceivable glaucoma.

\subsection{Computer-Based Generic Methods to Detect Glaucoma}

3.2.1. Fundus Image Analysis. One of the complex retinal imaging procedures to detect glaucoma is using fundus camera [13, 14]. A digital fundus camera equipped with a low-light microscope can record images of the entire retina and the posterior pole of the eye. The circular area termed as papilla is responsible for the connection of optic nerve to retina [15]. Disc region surrounding the lighter area is termed as cutting area. The disc and cup diameter of fundus and OCT are shown in Figure 3.

Singh et al. [17] extract the green channel so one can extract the cup and disc area from the fundus image. The green channel contains most of the information needed to 


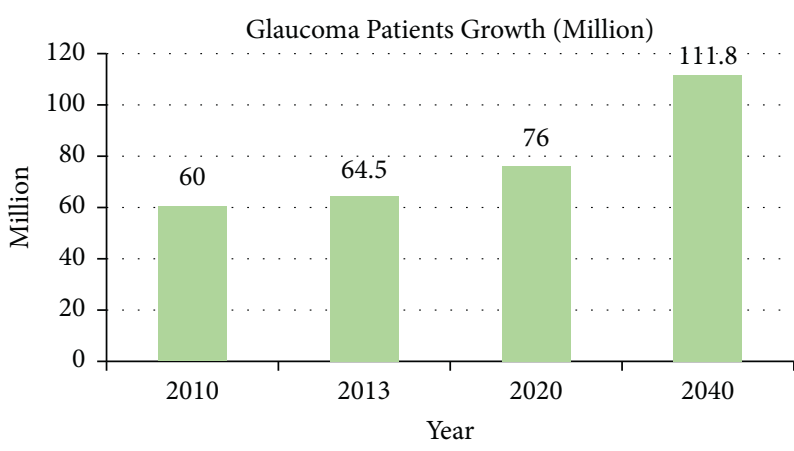

FIGURE 1: Expected rise in glaucoma patients across the world [4].

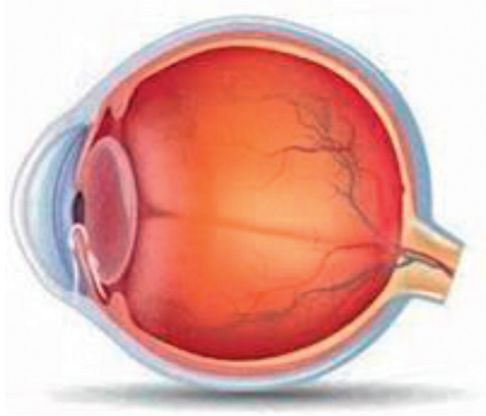

NORMAL

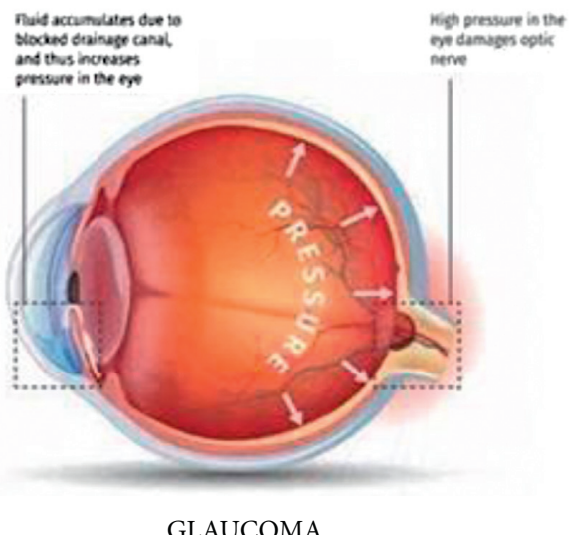

GLAUCOMA

Figure 2: Normal vs. glaucoma disease [5].

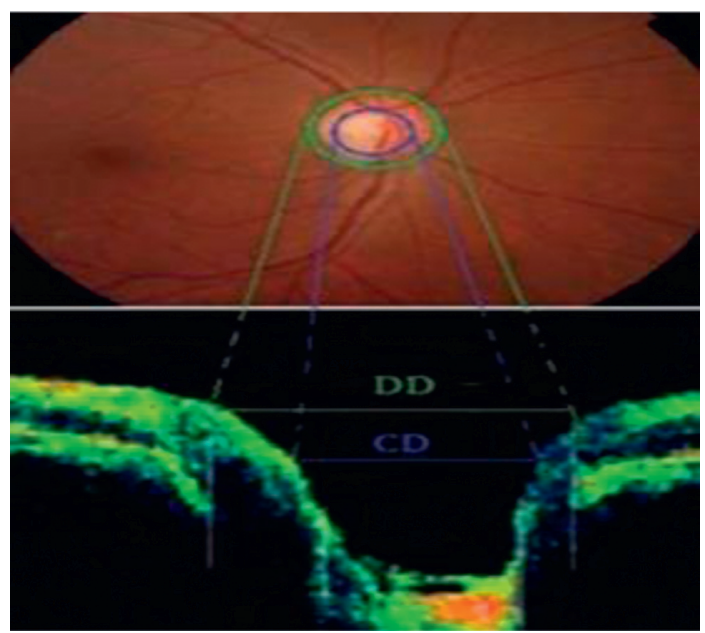

Figure 3: Disc diameter and cup diameter in fundus and an OCT image [16].

identify the center of disc. Select the Kaiser window for all lines and maximum values of the image that takes the $X$ coordinate of the intervertebral disc into account. However, this type of method relies only on the intensity of the pixels to locate the disk center. If other shiny artefacts like exudate and PPA are present, they will not give accurate results. Ahmad et al. [18] employed morphological operations for the extraction of optic disc in fundus images. Extracted cup and disc region is further used to calculate cup-to-disc ratio (CDR). The relationship between the boundary regions of the retina is also extracted from the features to assist with the detection of glaucoma patients. The credibility of established system was tested on small dataset on 80 fundus images and received a 97.5\% accuracy rate. Panda et al. [19] used a different technique to detect the disc center. In order to detect symmetrical lines for considering the properties of thick blood vessels, morphological operation was engaged. The second step involves the detection of optic disc center by counting the highest vessel component. In the third step, the author finalizes the exact location of optic disc center by concluding a local symmetry line along with radius. This approach is verified on nine publicly available datasets. Nevertheless, the proposed approach is not able to perform well on low-quality images or if the number of blood vessels is not sufficient. Fraga et al. [20] offered a method for the optic disc segmentation by introducing different stages. Retinex algorithm was used to normalize the retinal images to increase the process reliability and to diminish the disparity variability. The technique achieved $100 \%$ of optic disc localization. However, this approach did not involve pathologic retinal images that are affecting the optic disc overall accuracy. Salam et al. [21] proposed a unique method to classify fundus images into three categories: glaucoma patients, suspicious specimens, and nonglaucoma images. The method was tested on a local dataset containing 100 fundus images of 26 glaucoma patients and 74 nonglaucoma 
patients. Haleem et al. [22] proposed a methodology for segmenting and localizing the optic disc using vasculatures convergence and weighted feature maps. They have used the RIMONE and SLO datasets and obtained an average accuracy of $93.9 \%$. The authors take the test images and minimize the distance between the normal profiles of future maps by estimating the contour and in training set by calculating the mean of the images. Table 1 shows analysis for glaucoma detection using fundus images.

\subsubsection{Optical Coherence Tomography (OCT) Image Analysis.} OCT is a revolutionary imaging approach that is extensively used by ophthalmologists worldwide. OCT images provide detailed information about the layers of the retina. Optical coherence tomography (SD-OCT) is often used to improve retinal assessment measures [27]. The image affected by glaucoma has a larger cup diameter than the normal OCT image, as presented in Figure 4.

Siesky et al. [23] inspected progression of open angle glaucoma within 5 years of time. A total of 112 open-angle glaucoma patients were examined after every six months for the period of 5 years. Different perimetry devices like visual field, OCT, and Heidelberg retinal tomography were considered to observe the structural changes in eyes. Their findings concluded that both RNFL and ONH were associated with practical glaucomatous progression and visual field lost in patients during 5 years of examination. Rao et al. [24] tested 46 control and 61 glaucoma eyes. By measuring the thickness, the functional ability of glaucoma diagnosis was compared. They concluded that SDOCT is more suitable for measuring thickness. Gopinath et al. proposed a new method [25]. OCTA (Optical Coherence Tomography Angiography) imaging was used for glaucoma diagnosis. Capillary density (CD) and RNFL thickness are two estimated features that classify OCTA images as glaucoma or nonglaucoma images. After the capillary network is identified as a region of interest (ROI), measure the CD of eight sectors. The CD and RNFL thickness values of the evaluation parameter glaucoma detection system are lower than normal OCTA images in glaucoma patients. Nieves-Moreno et al. proposed an algorithm for detecting glaucoma on SD-OCT images of the retina [26]. Abdel-Hamid [28] proposed an automated glaucoma detection device primarily based on the frequency area discrete wavelet transform (DWT) of preprocessed OCT images. The noise of the image is removed by using median filter. After domain wavelet transform decomposition, the wavelet coefficients are the attributes extracted inside the frequency area.

3.2.3. Databases. Distinctive globally and locally accessible fundus and OCT datasets are accumulated for healthy and glaucoma patients. More information such as the names of the datasets, the images available in each dataset, the provision of the datasets, and the technology applied to them is depicted in Table 2. The HRF dataset contains 30 images including 15 healthy and 15 glaucomatous images with a resolution of $3504 * 2336$. Pathan et al. used the HRF dataset in a real-time detection system and achieved a precision of 96.7\% [29]. The Drishti database contains 101 images with a resolution of $2049 * 1757$, which are used for analyzing glaucoma. Its source is the publicly accessible Aravind Eye Hospital in India. Patil et al. [30] used the Drishti dataset to classify retinal features and obtained an accuracy of 97.4. The glaucoma DB dataset comprised of 452 images with a resolution of $1504 * 1000$ for analysis of glaucoma. This will contain different features like hybrid features, optic disk, and classification to classify glaucoma [31].

\subsubsection{Evaluation of OCT and Fundus for Glaucoma} Detection. An evaluation among fundus and OCT images for differentiating between ordinary and glaucoma sufferers is provided for further amplification. As shown in Table 1 and Figure 5 below, various methods in these two categories have been proposed and discussed. The researchers use various algorithms such as the Otsu method, the area growth algorithm, the climbing algorithm, the FCM clustering algorithm, and the optical pan segmentation method to segment the optic disc area and calculate the CDR value in the fundus image. In order to use OCT to assess glaucoma, various algorithms are provided. In order to segment the retinal nerve fiber and retinal pigment epithelium layer, segmentation algorithm is used. This layer-based segmentation allows to calculate the CDR value after segmenting optic disc and cup area. The CDR measurement of OCT imaging has been shown to be closer to the clinical outcomes of glaucoma patients. Nithya et al. [32]. A comparison was performed between fundus and OCT images to determine performance error. From experiment analysis, it is clearly indicated that glaucoma can be diagnosed more efficiently with OCT images. Due to the glaucoma, the deformity occurs in the cup-shaped area and can be seen more precisely on the OCT image, as it provides information about the retinal layer.

\section{CNN-Based Methods to Detect Glaucoma}

In recent years, deep learning technology has improved the technical level of classification, segmentation, and recognition of objects in medical and ophthalmological images; the summary of these is presented in Table 3 further in this section. As compared to traditional machine learning approaches that rely heavily on expert domain knowledge to employ functions, $\mathrm{CNN}$ is a network that maximizes the network capacity by learning functions to move between healthy and infected images. There are a variety of CNN architectures designed for image segmentation as well as classification. Each of these architectures is different in terms of number and size of functions, how these functions will be connected, and what would be the entire depth of the network. This is because different architecture is suited for different problems as per the nature of problem and it is challenging to know the priority of network, which architecture would be the right choice for a given task. The best way to make the decision in these circumstances is empirical testing. 
TABLE 1: Review of OCT and fundus imaging for glaucoma diagnosis.

\begin{tabular}{|c|c|c|c|c|c|}
\hline Year & $\begin{array}{l}\text { Image } \\
\text { modality }\end{array}$ & Features & Method & Dataset & Accuracy \\
\hline 2016 & Fundus & $\begin{array}{l}\text { Optic disc, cup-to-disc ratio } \\
{[17]}\end{array}$ & $\begin{array}{l}\text { Wavelet feature extraction } \\
\text { score normalization }\end{array}$ & Local database, 63 images & $94.7 \%$ \\
\hline 2017 & Fundus & $\begin{array}{l}\text { Optic disc, vessel map, } \\
\text { vessel component [19] }\end{array}$ & $\begin{array}{l}\text { Morphological operation for } \\
\text { glaucoma detection }\end{array}$ & $\begin{array}{l}10 \text { publicly available datasets; } \\
\text { only HRF provides center } \\
\text { ground truth }\end{array}$ & $99.49 \%$ \\
\hline 2016 & Fundus & $\begin{array}{l}\text { CDR, texture feature, color } \\
\text { moments, intensity [21] }\end{array}$ & $\begin{array}{c}\text { Wavelet, multiwavelet binary } \\
\text { pattern, Grey cooccurrence } \\
\text { matrix }\end{array}$ & $\begin{array}{l}50 \text { fundus images taken from } \\
\text { local dataset }\end{array}$ & $87 \%$ \\
\hline 2016 & Fundus & $\begin{array}{l}\text { Optic disc, CDR, regional } \\
\text { image feature [22] }\end{array}$ & Gaussian filter bank, & RIMONE, SLO & $93.9 \%$ \\
\hline 2020 & OCT & $\begin{array}{l}\text { Disc area, cup area, CDR, } \\
\text { RNFL thickness [23] }\end{array}$ & $\begin{array}{c}\text { Hazard models along with } \\
\text { OCT }\end{array}$ & Private & $\begin{array}{c}\text { Disc area: } 0.008(p \\
\text { value); RNFL thickness } \\
0.003 \text { ( } p \text { value })\end{array}$ \\
\hline 2017 & SDOCT & GCLIP thickness [24] & Macula by SAP, GCA, Z-test & $\begin{array}{c}\text { Private: } 127 \text { eyes images of } 80 \\
\text { participants }\end{array}$ & $75,0.65$ \\
\hline 2016 & OCTA & $\begin{array}{l}\text { RNFL thickness, capillary } \\
\text { density [25] }\end{array}$ & Layer segmentation method & Private dataset: 67 images & $94 \%$ \\
\hline 2018 & SDOCT & $\begin{array}{c}\text { Inner macular layer } \\
\text { thickness, inner retinal layer } \\
\text { thickness [26] }\end{array}$ & Spectralis OCT & $\begin{array}{l}\text { Private: } 148 \text { eyes of patients } \\
\text { (Spain and Belgium Hospital) }\end{array}$ & $\begin{array}{l}42.2 \% \\
88.9 \%\end{array}$ \\
\hline
\end{tabular}

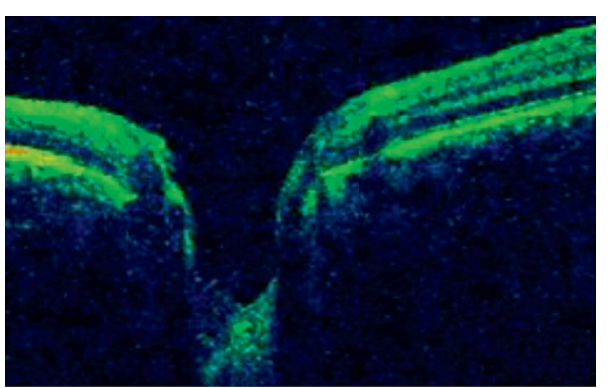

(a)

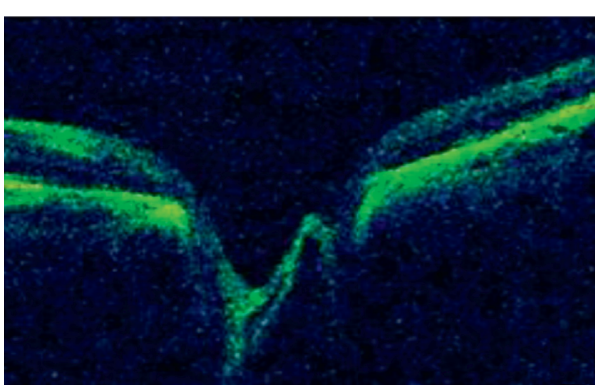

(b)

Figure 4: An OCT image. (a) Small cup-to-disc diameter ratio in the normal subject. (b) Larger cup-to-disc diameter is observed in the glaucoma-affected image [16].

TABLE 2: Datasets available for glaucoma detection in OCT and fundus imaging.

\begin{tabular}{|c|c|c|c|c|c|c|c|c|}
\hline No. & Dataset name & Images & Source & Resolution & $\begin{array}{c}\text { Type of retinal } \\
\text { diseases }\end{array}$ & $\begin{array}{c}\text { Field of } \\
\text { view } \\
(\mathrm{FOV})\end{array}$ & Availability & Links \\
\hline 1 & EyePACS 1 & 9963 & $\begin{array}{c}\text { EyePACS Program } \\
\text { (DR affected } \\
\text { patients) }\end{array}$ & $2592 \times 1224$ & $\begin{array}{l}\text { Glaucoma, } \\
\text { cataract }\end{array}$ & 45 & Public & $\begin{array}{c}\text { https://www. } \\
\text { medicmind.tech/retinal- } \\
\text { image-databases }\end{array}$ \\
\hline 2 & $\begin{array}{l}\text { High-Resolution } \\
\text { Fundus (HRF) }\end{array}$ & 30 & $\begin{array}{c}\text { Canon CR-1 } \\
\text { Fundus Camera }\end{array}$ & $3504 \times 2336$ & Glaucoma & 45 & Public & $\begin{array}{c}\text { https://www5.cs.fau.de/ } \\
\text { research/data/fundus- } \\
\text { images/ }\end{array}$ \\
\hline 3 & Drishti & 101 & $\begin{array}{c}\text { Aravind Eye } \\
\text { Hospital, Madurai, } \\
\text { India }\end{array}$ & $2049 \times 1757$ & Glaucoma & 45 & Public & $\begin{array}{l}\text { https://cvit.iiit.ac.in/ } \\
\text { projects/mip/drishti-gs/ } \\
\text { mip-dataset2/enter.php }\end{array}$ \\
\hline 4 & ORIGA & 650 & $\begin{array}{l}\text { SiMES, Fundus } \\
\text { Camera }\end{array}$ & $3072 \times 2048$ & Glaucoma & 50 & Public & $\begin{array}{c}\text { https://www. } \\
\text { medicmind.tech/retinal- } \\
\text { image-databases }\end{array}$ \\
\hline 5 & GlaucomaDB & 452 & Fundus database & $1504 \times 1000$ & Glaucoma & 45 & Public & $\begin{array}{c}\text { http://biomisa.org/ } \\
\text { glauco\%20m\%20adb/ }\end{array}$ \\
\hline
\end{tabular}




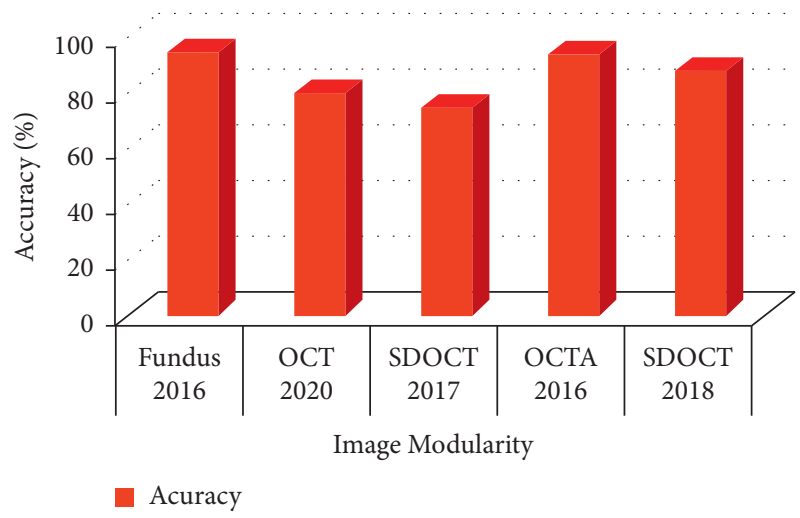

FIgURE 5: Comparison of image modulation versus accuracy (yearwise).

TABLE 3: Review of glaucoma diagnosis using CNN.

\begin{tabular}{|c|c|c|c|c|c|}
\hline Year & Algorithm & Feature & Method & Dataset & Accuracy \\
\hline 2016 & Deep CNN & $\begin{array}{c}\text { Optic disc } \\
\text { segmentation }\end{array}$ & $\begin{array}{l}\text { CNN using } 5 \text { layers with VGG- } \\
19 \text { network }\end{array}$ & DRIONS, RimOneR3 & F-score: 96 \\
\hline 2017 & $\mathrm{CNN}$ & $\begin{array}{l}\text { Optic cup and disc } \\
\text { segmentation }\end{array}$ & Sampling methodology & Drishti-GS1 & Accuracy: $94.10 \%$ \\
\hline 2017 & $\mathrm{CNN}$ & Disc segmentation & CNN with 7 layers & Drive & $\begin{array}{c}\text { Accuracy: } \\
\text {-Sensitivity: } 87\end{array}$ \\
\hline 2017 & $\begin{array}{l}\text { Ant colony } \\
\text { optimization }\end{array}$ & $\begin{array}{l}\text { Optic cup } \\
\text { segmentation }\end{array}$ & Ant colony optimization & RimoneR3 & $\begin{array}{c}\text { Accuracy: -AUC: } \\
0.7957\end{array}$ \\
\hline 2017 & DCNN & $\begin{array}{l}\text { Optic cup and disc } \\
\text { segmentation }\end{array}$ & $\begin{array}{c}\text { U-Net convolutional neural } \\
\text { network }\end{array}$ & Drishti-GS & 2017 \\
\hline 2018 & $\mathrm{CNN}$ & $\begin{array}{l}\text { Optic disc } \\
\text { segmentation }\end{array}$ & Two-branch CNN & Private & Accuracy: 81.69 \\
\hline 2018 & $\begin{array}{c}\text { Ensemble } \\
\text { learning CNN }\end{array}$ & $\begin{array}{l}\text { Optic disc } \\
\text { segmentation }\end{array}$ & $\mathrm{CNN}$ using CDR ratio & Rimone & Accuracy: 81.69 \\
\hline 2018 & DCNN & Fundus photography & Cross Entropy Inception V3 & $\begin{array}{l}\text { Private (Kin's Eye Hospital, } \\
\text { South Korea }\end{array}$ & Accuracy: 87.9 \\
\hline 2018 & $\mathrm{CNN}$ & ONH fundus images & GON in fundus photographs & $\begin{array}{l}\text { Private (the ADAGES new study } \\
\text { and Alabama, California) }\end{array}$ & $\begin{array}{c}\text { Accuracy: -AUC: } \\
0.91\end{array}$ \\
\hline 2018 & DCNN & $\begin{array}{l}\text { Optic cup and disc } \\
\text { segmentation }\end{array}$ & $\begin{array}{l}\text { Morphometric features for } \\
\text { CNN multistage model }\end{array}$ & Drishti-GS1 Rimone & Accuracy: $88.9 \%$ \\
\hline 2018 & $\mathrm{CNN}$ & $\begin{array}{l}\text { Optic disc } \\
\text { segmentation }\end{array}$ & $\begin{array}{c}\text { Entropy sampling, Ensemble } \\
\text { learning }\end{array}$ & Drishti-GS & Accuracy: $91.9 \%$ \\
\hline 2019 & Joint RCNN & $\begin{array}{l}\text { Optic cup and disc } \\
\text { segmentation }\end{array}$ & $\begin{array}{c}\text { Generative adversarial network } \\
\text { GL-Net }\end{array}$ & Drishti-GS1 Origa & $\begin{array}{l}\text { Accuracy: -AUC: } \\
90.10\end{array}$ \\
\hline 2019 & $\mathrm{CNN}$ & Proposed deep CNN & CNN with 18 layers & $\begin{array}{c}\text { Private Kasturba medical college } \\
\text { India }\end{array}$ & Accuracy: 98\% \\
\hline 2020 & $\mathrm{CNN}$ & $\begin{array}{l}\text { Optic cup and disc } \\
\text { segmentation }\end{array}$ & CNN OverFeat and VGG-S & Private & Accuracy: $90.50 \%$ \\
\hline 2020 & DCNN & $\begin{array}{l}\text { Optic Cup and Disc } \\
\text { segmentation }\end{array}$ & Joint RCNN & Origa & Accuracy: $92.50 \%$ \\
\hline
\end{tabular}

Zilly et al. [33]. Using the CNN network with VGG-19, the specialized layer is expanded to segment the optic disc. A stereoscopic image is an image that uses multiangle images combined to represent a $3 \mathrm{D}$ model of the retina. They achieved a mean $F$-score of $96.5 \%$ and a mean error rate of 5 pixels with a maximum margin of 16 pixels. More than $50 \%$ of the images have an error rate of more than 5 pixels. However, the marginal error of the RIMONER3 image is greater than that of DRIONS dataset. This shows the need to improve edge detection, especially in the presence of exudates. Additionally, cross-validation will be a more powerful verification technique than simply sharing the dataset during training and testing. Tan et al. [34] presented a CNNbased sample methodology that reduces computational complexity. Output of the last layer is entered as input from the new layer. New type of Softmax classifier is used consistently for the output of all filters. It is then applied to the test image. This method accomplished $95.60 \%$ specificity, $94.10 \%$ accuracy, and sensitivity $92.30 \%$ on DRISHTI-GS1 dataset. However, to train a CNN using few images seems incredible. This study proposed a method to learn CNN when dataset is small. By having $\mathrm{CNN}$ trained on less data 
means less parameters can be reliably trained; therefore, more advancement in this method is needed for better accuracy. Orlando et al. [35]. After normalizing the image, a seven-layer $\mathrm{CNN}$ is used to segment the optic disc. CNN has been trained in back propagation and stochastic gradient descent. Softmax is used for the linear units of the sixth and first layers and the activation of the leaky rectifiers of the third and fifth layers is used. This method achieved a sensitivity of $87 \%$, a specificity of $99 \%$, and an overlap error rate of $62 \%$. Select the drive dataset to produce the results of the proposed method. However, the treatment of exudate and PPA is not discussed. Furthermore, the proposed method was tested on 20 images only. This small dataset is certainly not sufficient to guarantee the correction and accuracy of the overall results. Orlando et al. [36] used two different architectures VGG-S and OverFeat to develop a CNN model for automatic glaucoma detection. For confirmation from current CNN (OverFeat and VGG-S), pretrained architectures for images without fundus images are implemented. Preprocessing takes place first for these newly acquired fund images to improve quality; then, $\mathrm{ONH}$ segmentation is applied. The proposed method influences $76.30 \%$ and $71.80 \%$ as AUC values. However, the author did not perform extensive comparison with other state-of-the-art strategies as most of the methods assessment used their own private dataset.

Chai et al. [37] used the concept of ant colony for optic cup segmentation in order to determine glaucoma in an early stage. The reason to use ant colony because it is a probabilistic technique, which makes sure to find an optimal path that will minimize the need to find a path through graph. The approach obtained an average overlap error of $24.3 \%$. Nevertheless, the approach to the ant colony suffers from an important limitation, namely, the uncertainty at the time of convergence. How this was handled was not discussed in the paper too. Zilly et al. [33] presented a CNN twofold structure. Segmented optics as input the disc was escalated to the CNN model and the entire CNN model was fed with the whole image. Likewise the concatenation was performed on the CNN model and a fully connected layer was used for classification. This method reaches $81.69 \%$ as a classification precision for the Beijing Tongren China Hospital dataset. Jiang et al. [38] improved their existing work by applying CNN to extract optic cup for diagnosis of glaucoma. To get around the challenge of using CNN (which requires a large dataset) discussed above, the authors incorporated a colearning approach. With a marginal error of 11.8 pixels, an average $F$ value of $84.75 \%$ was obtained. In terms of the cup, this is a great achievement, yet further improvements in performance and accuracy are possible. Furthermore, the authors did not test their method on other publicly accessible datasets in order to compare it to other methods; therefore, they were unable to validate its robustness. Jiang et al. [39] use the DCNN multilabel model with GL-Net. This method achieved $90.50 \%$ and $97.10 \%$ of the $F 1$ values for the optic disc and cup, respectively. Ahan et al. [40] used CNN for diagnosis of glaucoma. Two convolutional and hidden layers along with max pooling were applied. ReLU served as an activation function. The weights were assigned with Xavier initializer. The proposed model was tested on small dataset collected from Kim's Eye Hospital. The total 1542 images were collected from which 756 were glaucomatous and 786 were nonglaucomatous. This method achieved 87.9 accuracy by using fundus photography. However, they have used a small dataset and therefore unable to verify the robustness of their proposed solution. Jiang et al. [41] diagnose glaucoma by segmenting the optic cup and disc using the CNN algorithm. The proposed end-to-end algorithm is called the ordinary RCNN. Porous folding plays an important role in improving performance. Two datasets named SECS and ORIGA evaluate this approach. In the dataset, AUCs of $90.10 \%$ and $85.40 \%$ were achieved. Christopher et al. [42] used a large $\mathrm{ONH}$ oblique fundus image database to evaluate several different CNN architectures based on the accuracy of glaucoma diagnosis. They achieved an AUC of 0.97 for GON eyes with moderate-to-severe loss of function. However, for the current study, the longitudinal section of the data is not taken into account, but the dataset is used to train the model, and each image is viewed separately. The authors of [43] concluded that the standard CNN deep learning model is not designed to handle and compute the longitudinal data. For the OC (Optical Cutting) and OD (Optical Disc) segmentation, an improved U-Net Convolutional Neural Network technology based on deep learning is proposed. With the help of deep learning, U-Net and CNN technologies have a very simple framework that takes much less time to implement and predict, which is much better than the most advanced technologies. The proposed method is implemented on the RIM-ONE and DRISHTI-GS datasets that provide RIM-ONE with Dice 0.82 and IOU 0.69 performance metrics, and DRISHTI-GS provides Dice 0.85 and IOU 0.75 performance metrics. The paper does not report accuracy of glaucoma detection. Raghavendra et al. [4] detect glaucoma through convolutional neural network. The proposed model consists of three steps with 15 layers CNN. Drishti dataset is used to evaluate the proposed model. This method obtained $89.4 \%$ accuracy. Despite the fact that deep $\mathrm{CNN}$ requires a huge number of datasets for training, it is feasible to gain improved accuracy by training the model with a big number of frames.

Raghavendra et al. [44] use CNN algorithm to diagnose glaucoma. The pixels of the input image are allocated hierarchically to estimate the background image. The private dataset is used to train the CNN algorithm from scratch. This model is most suitable for a large number of dataset images. The overall accuracy rate reached $98 \%$. However, due to the large number of images required for training, this method cannot be used for small datasets. Mookiah et al. [33] use histogram and random transformation for fundus imaging to diagnose glaucoma. In addition to processing, properties such as frequency spectrum and wavelet transformation can also be extracted from the image. The SVM classifier later uses this information to classify the image as normal or glaucoma. The method was tested on a small private dataset with 60 images. It achieved an accuracy of 95\%. For clinical implementation, the proposed method should be evaluated using a large dataset with characteristics from the domain. 


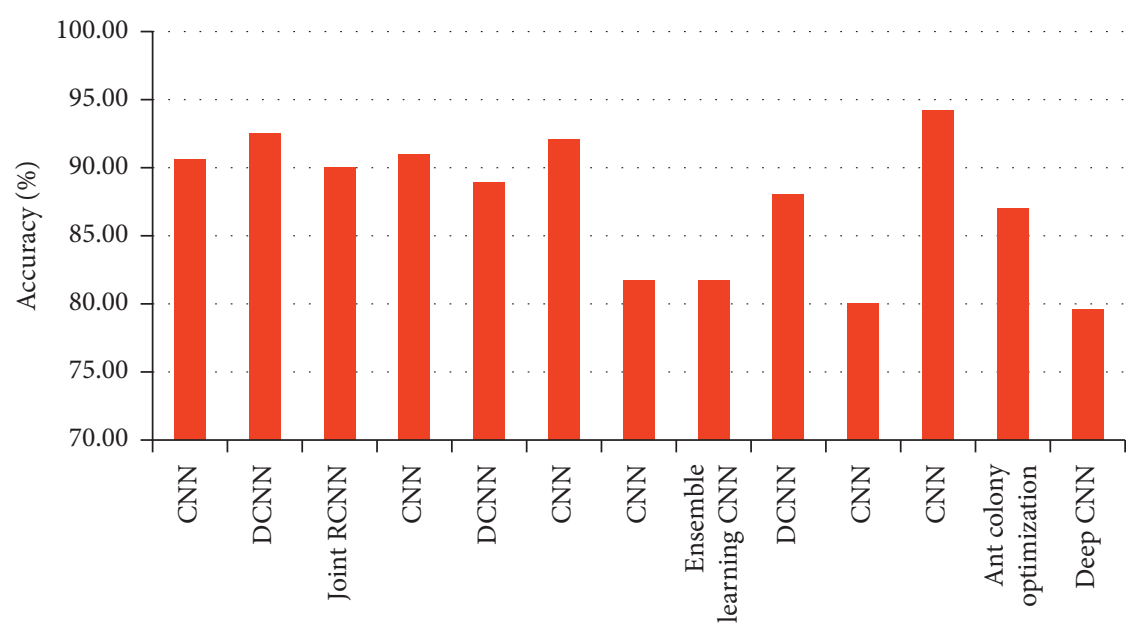

Algorithms

Accuracy

Figure 6: Conventional neural network algorithms versus accuracy.

Julian et al. [45] use convolutional neural networks for OD and OC segmentation. The researchers introduced technologies based on community learning. Convolution filter framework are designed by using filter points. Use the output of the filter to train the final flexible logistics classifier. The output of the classifier is divided by the intersection of the graph and the convex envelope. Use OD and OC to calculate the segmented CDR. The method is evaluated using the Drishti dataset, which consists of 101 images. The three coefficients obtained by dividing the OD and OC are $97 \%$ and $87 \%$, respectively. However, for OD classification, this approach works well. The topic of optimizing network parameters in order to decrease computing effort is a challenge. The review of glaucoma diagnosis using $\mathrm{CNN}$ is presented in Table 3 and Figure 6.

\section{Conclusion}

The development of convolution algorithms for neural networks has opened up the possibility of improving the diagnosis of glaucoma in the test environment and in clinical practice. Not only does the CNN algorithm reduce the tedious work of manually reviewing annotated images, it can also accurately identify glaucoma in OCT and fundus photos, which can improve its usefulness in high-pressure environments with limited resources. The algorithm can also identify other features on the OCT B-scan that are important in diagnosing glaucoma or its progression. However, the performance of the $\mathrm{CNN}$ algorithm is heavily influenced by the data and image quality used for training and testing. It is important to use a more representative dataset that includes multiethnic populations and related pathologies of the retina and optic nerve head. Additionally, CNN may perform better on test images obtained from the same camera used for the training set. Hence, more training and validation are needed to support the data obtained on different platforms. In addition, when applied to images taken in real-world conditions where the prevalence of glaucoma is much lower, it is particularly important to ensure that $\mathrm{CNN}$ is highly specific to glaucoma. Future research should apply these algorithms under real-world conditions to see if they perform well in clinical and screening scenarios when compared to human practitioners.

Previous studies evaluated the burden of vision loss in Pakistan in terms of prevalence of eye diseases alone. We used both prevalence and yield methods to investigate the vision loss burden in our study. This enabled us to compare the burden of vision loss in Pakistan with those in other countries and other diseases in a more detailed manner. There is no medication for glaucoma still, and vision loss is irretrievable; consequently, molecular diagnostics for predictive assessment and early interference is essential to decrease the influence of visual mutilation and eventually blindness. To accomplish this goal, the requirement is to exemplify all subtypes of glaucoma at the molecular level and recognize loci/genes contributing to this ophthalmic disorder in diverse populaces.

\section{Data Availability}

The data used to support the findings of this study are available from the corresponding author upon request.

\section{Conflicts of Interest}

The authors declare that they have no conflicts of interest regarding the publication of this paper.

\section{References}

[1] A. Auraaen, L. Slawomirski, and N. Klazinga, The Economics of Patient Safety in Primary and Ambulatory Care: Flying Blind, OECD Publishing, 2018.

[2] S. Kumpatla, H. Kothandan, S. Tharkar, and V. Viswanathan, "The costs of treating long-term diabetic complications in a developing country: a study from India," Journal of the 
Association of Physicians of India, vol. 61, no. 1, pp. 102-109, 2013.

[3] A. L. Pelletier, L. Rojas-Roldan, and J Coffin, "Vision loss in older adults," American Family Physician, vol. 94, no. 3, pp. 219-226, 2016.

[4] U. Raghavendra, H. Fujita, S. V. Bhandary, A. Gudigar, J. H. Tan, and U. R. Acharya, "Deep convolution neural network for accurate diagnosis of glaucoma using digital fundus images," Information Sciences, vol. 441, pp. 41-49, 2018.

[5] Please provide complete reference details.

[6] B. Hassan, R. Ahmed, B. Li, A. Noor, and Z. U. Hassan, "A comprehensive study capturing vision loss burden in Pakistan (1990-2025): findings from the Global Burden of Disease (GBD) 2017 study," PloS one, vol. 14, no. 5, p. e0216492, 2019.

[7] L. Khan, M. Ali, M. Qasim, F. Jabeen, and B. Hussain, "Molecular basis of glaucoma and its therapeutical analysis in Pakistan: an overview," Biomedical Research and Therapy, vol. 4, no. 3, pp. 1210-1227, 2017.

[8] T. Khalil, M. U. Akram, S. Khalid, S. H. Dar, and N. Ali, “A study to identify limitations of existing automated systems to detect glaucoma at initial and Curable Stage," International Journal of Imaging Systems and Technology, vol. 31, no. 3, pp. 1155-1173, 2021.

[9] Y. H. Kwon, J. H. Fingert, M. H. Kuehn, and W. L. M. Alward, "Primary open-angle glaucoma," New England Journal of Medicine, vol. 360, no. 11, pp. 1113-1124, 2009.

[10] C. Wright, M. A. Tawfik, M. Waisbourd, and L. J. Katz, "Primary angle-closure glaucoma: an update," Acta Ophthalmologica, vol. 94, no. 3, pp. 217-225, 2016.

[11] S. Munkwitz, A. Elkarmouty, E. M. Hoffmann, N. Pfeiffer, and H. Thieme, "Comparison of the iCare rebound tonometer and the Goldmann applanation tonometer over a wide IOP range," Graefes Archive for Clinical and Experimental Ophthalmology, vol. 246, no. 6, pp. 875-879, 2008.

[12] M. S. Haleem, L. Han, J. Van Hemert, and B. Li, "Automatic extraction of retinal features from colour retinal images for glaucoma diagnosis: a review," Computerized Medical Imaging and Graphics: The Official Journal of the Computerized Medical Imaging Society, vol. 37, no. 7-8, pp. 581-596, 2013.

[13] A. Mitani, A. Huang, S. Venugopalan et al., "Detection of anaemia from retinal fundus images via deep learning," Nature Biomedical Engineering, vol. 4, no. 1, pp. 18-27, 2020.

[14] J. I. Orlando, E. Prokofyeva, M. Del Fresno, and M. B. Blaschko, "An ensemble deep learning based approach for red lesion detection in fundus images," Computer Methods and Programs in Biomedicine, vol. 153, pp. 115-127, 2018.

[15] M. Naveed, A. Ramzan, and M. U. Akram, "Clinical and technical perspective of glaucoma detection using OCT and fundus images: a review," in 2017 1st International Conference on Next Generation Computing Applications (NextComp), pp. 157-162, IEEE, 2017.

[16] Biomisa Biomedical Image/Signal Analysis (BIOMISA) Research Lab, National University of Science and Technology, Islamabad, Pakistan, 2017, http://biomisa.org/glaucomadb/.

[17] A. Singh, M. K. Dutta, M. ParthaSarathi, V. Uher, and R. Burget, "Image processing based automatic diagnosis of glaucoma using wavelet features of segmented optic disc from fundus image," Computer Methods and Programs in Biomedicine, vol. 124, pp. 108-120, 2016.

[18] H. Ahmad, A. Yamin, A. Shakeel, S. O. Gillani, and U. Ansari, "Detection of glaucoma using retinal fundus images," in Proceedings of the 2014 International conference on robotics and emerging allied technologies in engineering (iCREATE), pp. 321-324, IEEE, Islamabad, Pakistan, April 2014.

[19] R. Panda, N. B. Puhan, and G. Panda, "Robust and accurate optic disk localization using vessel symmetry line measure in fundus images," Biocybernetics and Biomedical Engineering, vol. 37, no. 3, pp. 466-476, 2017.

[20] A. Fraga, N. Barreira, M. Ortega, M. G. Penedo, and M. J. Carreira, "Precise segmentation of the optic disc in retinal fundus images," in International Conference on Computer Aided Systems Theory, pp. 584-591, Springer, Berlin, Heidelberg, 2011.

[21] A. A. Salam, M. U. Akram, A. Arouj et al., "Benchmark data set for glaucoma detection with annotated cup to disc ratio," in Proceedings of the 2017 International Conference on Signals and Systems (ICSigSys), pp. 227-233, IEEE, Bali, Indonesia, May 2017.

[22] M. S. Haleem, L. Han, J. Van Hemert et al., "Regional image features model for automatic classification between normal and glaucoma in fundus and scanning laser ophthalmoscopy (SLO) images," Journal of Medical Systems, vol. 40, no. 6, p. 132, 2016.

[23] B. Siesky, S. M. Wentz, I. Januleviciene et al., "Baseline structural characteristics of the optic nerve head and retinal nerve fiber layer are associated with progressive visual field loss in patients with open-angle glaucoma," PloS one, vol. 15, no. 8, p. e0236819, 2020.

[24] H. L. Rao, Z. S. Pradhan, R. N. Weinreb et al., "Optical coherence tomography angiography vessel density measurements in eyes with primary open-angle glaucoma and disc hemorrhage," Journal of Glaucoma, vol. 26, no. 10, pp. 888-895, 2017.

[25] K. Gopinath, J. Sivaswamy, and T. Mansoori, "Automatic glaucoma assessment from angio-OCT images," in Proceedings of the 2016 IEEE 13th International Symposium on Biomedical Imaging (ISBI), pp. 193-196, IEEE, Prague, Czech Republic, April 2016.

[26] M. Nieves-Moreno, J. M. Martínez-de-la-Casa, M. P. Bambo et al., "New normative database of inner macular layer thickness measured by spectralis OCT Used as reference standard for glaucoma detection," Translational vision science \& technology, vol. 7, no. 1, p. 20, 2018.

[27] J. Wang and L. R. Yin, "The application of enhanced depth imaging spectral-domain optical coherence tomography in macular diseases," Journal of Ophthalmology, vol. 2020, pp. 1-7, 2020.

[28] L. Abdel-Hamid, "Glaucoma detection from retinal images using statistical and textural wavelet features," Journal of Digital Imaging, vol. 33, no. 1, pp. 151-158, 2020.

[29] S. Pathan, P. Kumar, R. M. Pai, and S. V. Bhandary, "Automated segmentation and classifcation of retinal features for glaucoma diagnosis," Biomedical Signal Processing and Control, vol. 63, p. 102244, 2021.

[30] N. Patil, P. N. Patil, and P. V. Rao, "Automatic detection of optic disc using distance regularized level-set segmentation for glaucoma screening system," International Journal of Intelligent Unmanned Systems, vol. 10, no. 1, pp. 168-178, 2021.

[31] R. Nithya and N. Venkateswaran, "Analysis of segmentation algorithms in colour fundus and OCT images for glaucoma detection," Indian Journal of Science and Technology, vol. 8, no. 24, p. 1, 2015.

[32] K. K. Maninis, J. Pont-Tuset, P. Arbeláez, and L. Van Gool, "Deep retinal image understanding," in Proceedings of the International conference on medical image computing and 
computer-assisted intervention, pp. 140-148, Springer, Cambridge, UK, September 1999.

[33] J. Zilly, J. M. Buhmann, and D. Mahapatra, "Glaucoma detection using entropy sampling and ensemble learning for automatic optic cup and disc segmentation," Computerized Medical Imaging and Graphics, vol. 55, pp. 28-41, 2017.

[34] J. H. Tan, U. R. Acharya, S. V. Bhandary, K. C. Chua, and S. Sivaprasad, "Segmentation of optic disc, fovea and retinal vasculature using a single convolutional neural network," Journal of Computational Science, vol. 20, pp. 70-79, 2017.

[35] J. I. Orlando, E. Prokofyeva, M. del Fresno, and M. B. Blaschko, "Convolutional neural network transfer for automated glaucoma identification," in Proceedings of the 12th international symposium on medical information processing and analysis, vol. 10160, p. 101600U, December 2016.

[36] R. Arnay, F. Fumero, and J. Sigut, "Ant colony optimizationbased method for optic cup segmentation in retinal images," Applied Soft Computing, vol. 52, pp. 409-417, 2017.

[37] Y. Chai, L. He, Q. Mei, H. Liu, and L. Xu, "Deep learning through two-branch convolutional neuron network for glaucoma diagnosis," in International Conference on Smart Health, pp. 191-201, Springer, Cham, 2017.

[38] Y. Jiang, L. Duan, J. Cheng et al., "JointRCNN: a region-based convolutional neural network for optic disc and cup segmentation," IEEE Transactions on Biomedical Engineering, vol. 67, no. 2, pp. 335-343, 2019.

[39] J. M. Ahn, S. Kim, K. S. Ahn, S. H. Cho, K. B. Lee, and U. S. Kim, "A deep learning model for the detection of both advanced and early glaucoma using fundus photography," PloS one, vol. 13, no. 11, p. e0207982, 2018.

[40] Y. Jiang, N. Tan, and T. Peng, "Optic disc and cup segmentation based on deep convolutional generative adversarial networks," IEEE Access, vol. 7, pp. 64483-64493, 2019.

[41] M. Christopher, A. Belghith, C. Bowd et al., "Performance of deep learning architectures and transfer learning for detecting glaucomatous optic neuropathy in fundus photographs," Scientific Reports, vol. 8, no. 1, pp. 1-13, 2018.

[42] A. Sevastopolsky, "Optic disc and cup segmentation methods for glaucoma detection with modification of U-Net convolutional neural network," Pattern Recognition and Image Analysis, vol. 27, no. 3, pp. 618-624, 2017.

[43] O. Perdomo, V. Andrearczyk, F. Meriaudeau, H. Müller, and F. A. González, "Glaucoma diagnosis from eye fundus images based on deep morphometric feature estimation," in Computational Pathology and Ophthalmic Medical Image Analysis, pp. 319-327, Springer, Cham, 2018.

[44] M. R. K. Mookiah, U. R. Acharya, C. M. Lim, A. Petznick, and J. S. Suri, "Data mining technique for automated diagnosis of glaucoma using higher order spectra and wavelet energy features," Knowledge-Based Systems, vol. 33, pp. 73-82, 2015.

[45] T. Khalil, M. U. Akram, S. Khalid, S. H. Dar, and N. Ali, "A study to identify limitations of existing automated systems to detect glaucoma at initial and Curable Stage," International Journal of Imaging Systems and Technology, vol. 31, no. 3, pp. 1155-1173, 2021. 